\title{
MicroRNA-539 inhibits cell proliferation, colony formation and invasion in pancreatic ductal adenocarcinoma by directly targeting IGF-1R
}

\author{
YONGQUAN LIN ${ }^{1 *}$, LIHUA RONG $^{1 *}$, JINGRONG ZHAO $^{2}$, RONGHUI LIN ${ }^{3}$ and SHUHUA LI ${ }^{4}$ \\ ${ }^{1}$ Department of Emergency, Yidu Central Hospital of Weifang; ${ }^{2}$ Department of General Surgery, The 89th Hospital of \\ Chinese People's Liberation Army, Weifang, Shandong 262500; ${ }^{3}$ Health Clinics, Qingzhou Yanghe River Authority, Qingzhou, \\ Shandong 261021; ${ }^{4}$ Department of Emergency, Weifang People's Hospital, Weifang, Shandong 261000, P.R. China
}

Received December 22, 2017; Accepted May 15, 2018

DOI: $10.3892 / \mathrm{mmr} .2018 .9109$

\begin{abstract}
MicroRNAs (miRNAs) possess oncogenic and tumour-suppressive roles in the carcinogenesis and progression of pancreatic ductal adenocarcinoma (PDAC) by regulating the expression of numerous cancer-related genes. Thus, the investigation on the expression and roles of miRNAs in PDAC may facilitate the identification of novel and effective targets for the clinical diagnosis and treatment of patients with PDAC. miRNA-539 (miR-539) has been studied in multiple types of human cancer. However, its expression and potential biological function in PDAC remain unclear. In the current study, the expression level, clinical significance, roles and underlying molecular mechanism of miR-539 in PDAC. The present results demonstrated that miR-539 expression was downregulated in PDAC tissues and cell lines. A low miR-539 level was associated with TNM stage and lymph node metastasis of patients with PDAC. miR-539 overexpression induced a significant reduction in the proliferation, colony formation and invasion of PDAC cells. Insulin-like growth factor 1 receptor (IGF-1R) was confirmed as a direct target gene of miR-539 in PDAC. Further analysis indicated that IGF-1R was overexpressed in PDAC tissues. Notably, the mRNA expression of IGF-1R was negatively correlated with miR-539 levels in PDAC tissues. In addition, the recovered IGF-1R expression also partially counteracted the suppressive roles of miR-539 overexpression in PDAC cells. Overall, miR-539 may inhibit the aggressive
\end{abstract}

Correspondence to: Professor Shuhua Li, Department of Emergency, Weifang People's Hospital, 151 Guangwen Road, Weifang, Shandong 261000, P.R. China

E-mail: 1sh_weifang@yeah.net

${ }^{*}$ Contributed equally

Key words: pancreatic ductal adenocarcinoma, microRNA-539, proliferation, invasion, insulin-like growth factor 1 receptor, colony formation behaviour of PDAC by directly targeting IGF-1R and may serve as a novel therapeutic target for patients with this disease.

\section{Introduction}

Pancreatic cancer, the most malignant type of digestive system tumour, ranks as the seventh-most common cause of cancer-related deaths globally (1). Pancreatic ductal adenocarcinoma (PDAC), the main subtype of pancreatic cancer, accounts for approximately $90 \%$ of all pancreatic cancer cases (2). Despite a remarkable development in treatments and perioperative management, the prognosis of patients with PDAC remains poor. The median survival period and 5-year overall survival rate of patients with PDAC are approximately 6 months and less than 5\%, respectively (3). The poor therapeutic outcomes of PDAC patients are due to the late onset of presentation, metastasis and unresponsiveness to chemotherapy and radiation therapy (4). The pathogenesis of PDAC is influenced by various factors, including poor dietary habits, smoking, excessive drinking, long-term exposure to chemical carcinogens, diabetes mellitus and chronic pancreatitis $(5,6)$. However, the detailed mechanisms of the formation and progression of PDAC remain largely unknown. Therefore, the molecular mechanism of PDAC onset and development should be understood, and new therapeutic targets for the treatment of patients with this highly aggressive malignancy should be explored.

MicroRNAs (miRNAs) are noncoding and highly conserved short RNA molecules with 19 to 24 nucleotides implicated in gene regulation (7). miRNAs are involved in the regulation of their target genes by base pairing with the 3'-untranslated regions (3'-UTRs) of their target genes, thereby degrading mRNA and suppressing translation; thus, the expression levels of associated proteins are inhibited (8). miRNA dysregulation frequently occurs in numerous human malignancies, such as PDAC (9), melanoma (10), gastric cancer (11), colorectal cancer (12) and bladder cancer (13). Aberrantly expressed miRNAs play key roles in the initiation and progression of PDAC by improving oncogene expression or by downregulating the level of tumour suppressor genes (14-16). Hence, an in-depth understanding of miRNAs expression patterns and 
biological roles in PDAC may be advantageous to the development of miRNA-based targeted therapy, which may enhance the diagnosis, treatment and prognosis of patients with this fatal disease.

miR-539 has been studied in multiple types of human cancer (17-19). However, its expression and potential biological function in PDAC remain unclear. In our current study, we detected the expression level, clinical significance, roles and underlying molecular mechanism of miR-539 in PDAC.

\section{Materials and methods}

Tissue samples. Forty-five pairs of PDAC tissues and adjacent normal pancreatic tissues were collected from patients who received surgical resection at Yidu Central Hospital of Weifang between February 2014 and August 2016. All of the patients did not receive other treatments before surgery. This research was approved by the Ethics Committee of Yidu Central Hospital of Weifang (no. 2014036). The use of these tissue samples was approved by all of the patients before they participated in this project, and written informed consent was obtained from all of the patients.

Cell lines. Normal human pancreatic cell line (HPDE6c7) was acquired from American Type Culture Collection (Manassas, VA, USA). Four PDAC cell lines (Sw1990, Panc-1, Bxpc-3 and Aspc-1) from Shanghai Cell Bank of the Chinese Academy of Sciences (Shanghai, China) were grown in Dulbecco's modified Eagle's medium (DMEM) supplemented with $10 \%$ fetal bovine serum (FBS) and 1\% penicillin/streptomycin mixture (all from Gibco; Thermo Fisher Scientific, Inc., Waltham, MA, USA) and then maintained in a humidified atmosphere with $5 \% \mathrm{CO}_{2}$ at $37^{\circ} \mathrm{C}$.

Reverse transcription-quantitative polymerase chain reaction (RT-qPCR). Total RNA of tissue samples or cells was isolated with a TRIzol reagent (Invitrogen; Thermo Fisher Scientific, Inc.), in accordance with the manufacturer's instructions. Afterwards, the concentration of total RNA was detected using a NanoDrop 2000 (NanoDrop Technologies; Thermo Fisher Scientific, Inc., Pittsburgh, PA, USA). To quantify miR-539 level, total RNA was reversed transcription into complementary DNA (cDNA) with a TaqMan MicroRNA reverse transcription kit, and then quantitative PCR was conducted with a TaqMan MicroRNA PCR kit (all from Applied Biosystems; Thermo Fisher Scientific, Inc., Waltham, MA, USA) under Applied Biosystems ${ }^{\circledR}$ 7900HT Real-Time PCR system (Thermo Fisher Scientific, Inc.). To analyse IGF-1R mRNA expression, cDNA was synthesized from total RNA using a Primescript ${ }^{\mathrm{TM}}$ RT reagent kit. Subsequently, the cDNA was subjected into amplification with a SYBR Premix Ex Taq ${ }^{\mathrm{TM}}$ II kit (all from Takara Biotechnology Co., Ltd., Dalian, China). Relative miR-539 and IGF-1R mRNA expression was normalized to U6 snRNA and $\beta$-action, respectively. Data were analyzed using the $2^{-\Delta \Delta C q}$ method (20).

Cell transfection. miR-539 mimics and negative control miRNA mimics (miR-NC) were obtained from GenePharma (Shanghai, China). Insulin-like growth factor 1 receptor (IGF-1R) overexpression vector (pCMV-IGF-1R) and empty
pCMV vector were generated from Amspring Biological Technology Co., Ltd. (Changsha, China). For functional experiments, the cells were plated in 6-well plates 1 day prior to transfection. Cell transfection or cotransfection was carried out using Lipofectamine ${ }^{\circledR} 2000$ (Invitrogen; Thermo Fisher Scientific, Inc.) according to the manufacturer's protocol. The transfected cells were then cultured at $37^{\circ} \mathrm{C}$ in a humidified atmosphere with $5 \% \mathrm{CO}_{2}$, and the culture medium was replaced with fresh DMEM containing 10\% FBS at $6 \mathrm{~h}$ post transfection.

Cell Counting Kit (CCK)-8 and colony formation assays. CCK-8 assay was utilised to determine cell proliferative ability. For CCK-8 assay, the transfected cells were collected and seeded into 96 -well plates at a density of $3 \times 10^{3}$ cells per well with $100 \mu \mathrm{l}$ of the culture medium. The extent of proliferation was evaluated at $0,24,48$ and $72 \mathrm{~h}$ after incubation at $37^{\circ} \mathrm{C}$ in a humidified atmosphere with $5 \% \mathrm{CO}_{2}$. At each time point, $10 \mu \mathrm{l}$ of CCK-8 solution (Dojindo Molecular Technologies, Inc., Kumamoto, Japan) was added, and the cells were incubated for another $2 \mathrm{~h}$. Absorbance was detected at a wavelength of $450 \mathrm{~nm}$ by using an enzyme-linked immunosorbent assay plate reader (BioTek Instruments, Inc., Winooski, VT, USA).

Colony formation assay was carried out to examine the cell colony formation ability. The transfected cells were harvested and plated into 6 -well plates at a density of $1 \times 10^{3}$ cells per well. The plates were shaken to disperse the cells equally, and the cells were cultured in an incubator at $37^{\circ} \mathrm{C}$ for 7 days. On day 8 , the colonies were fixed with $100 \%$ methanol, stained with $0.5 \%$ crystal violet and rinsed in phosphate-buffered saline (PBS). The colonies were observed using a microscope, and the colonies containing $>50$ cells were counted.

Cell invasion assay. Transwell chambers with $8 \mu \mathrm{m}$ pores (BD Biosciences, San Jose, CA, USA) were applied to assess the cell invasive ability. The upper chambers were precoated with $100 \mu \mathrm{l}$ of diluted Matrigel ( $1 \mathrm{mg} / \mathrm{ml}$; BD Biosciences) and then incubated at $37^{\circ} \mathrm{C}$ for additional $1 \mathrm{~h}$. Afterwards, $1 \times 10^{5}$ transfected cells in FBS-free DMEM were plated into the upper chambers, and $500 \mu \mathrm{l}$ of DMEM containing 10\% FBS was added into the lower chambers. The cells were cultured at $37^{\circ} \mathrm{C}$ for $24 \mathrm{~h}$, and the cells remaining on the upper chambers were scraped off gently with cotton swabs. The invading cells were fixed with $100 \%$ methanol, stained with $0.5 \%$ crystal violet and washed with PBS. The invading cells were photographed and counted under an inverted microscope (CKX41; Olympus, Tokyo, Japan) in five randomly selected fields.

Bioinformatics prediction. TargetScan (http://www.targetscan. org/) and PicTar (http://pictar.mdcberlin.de/) were employed to predict the potential targets of miR-539. IGF-1R, a well-known oncogene, was predicted as a major highly conserved target of miR-539.

Luciferase reporter assay. Luciferase reporter plasmids, pMIR-IGF-1R-3'-UTR wild-type (Wt) and pMIR-IGF-1R-3'-UTR mutant (Mut), were designed, synthesised and confirmed by GenePharma. The cells were seeded into 24-well plates at a density of $6 \times 10^{4}$ cells per well 1 day prior to transfection. These cells were then transfected with miR-539 mimics or 
miR-NC and cotransfected with pMIR-IGF-1R-3'-UTR Wt or pMIR-IGF-1R-3'-UTR Mut by using Lipofectamine ${ }^{\circledR} 2000$, according to the manufacturer's instructions. Relative luciferase activities were measured at $48 \mathrm{~h}$ posttransfection with a dual-luciferase reporter assay kit (Promega Corporation, Madison, WI, USA) and normalised to that of Renilla activities.

Western blot analysis. Total protein of tissues or cells was lysed in a radioimmunoprecipitation assay lysis buffer, and the concentration of the total protein was detected with a bicinchoninic acid assay kit (all from Beyotime Institute of Biotechnology, Haimen, China). Equal amounts of protein were separated through $10 \%$ sodium dodecyl sulfate polyacrylamide gel electrophoresis and transferred to polyvinylidene fluoride membranes (EMD Millipore, Billerica, MA, USA). These membranes were blocked with $5 \%$ non-fat powdered milk in TBS-Tween-20 (TBST), incubated with primary antibodies overnight at $4^{\circ} \mathrm{C}$, washed with TBST thrice and further incubated with horseradish peroxidase-conjugated secondary antibody at room temperature for $1 \mathrm{~h}$. The protein signals were visualised using an enhanced chemiluminescence reagents (Pierce; Thermo Fisher Scientific, Inc.). Densitometric analysis was performed using Quantity One software version 4.62 (Bio-Rad Laboratories, Inc., Hercules, CA, USA). The primary antibodies used in this study include mouse anti-human IGF-1R monoclonal antibody (1:1,000 dilution; cat no. sc-462; Santa Cruz Biotechnology, Inc., Dallas, TX, USA) and mouse anti-human GAPDH monoclonal antibody $(1: 1,000$ dilution; cat no. sc-365062; Santa Cruz Biotechnology, Inc.).

Statistical analysis. Data were expressed as mean \pm standard deviation from at least three separate experiments. SPSS 19.0 (SPSS, Inc., Chicago, IL, USA) was used to perform statistical analysis. Qualitative data were analysed with chi-square test. Independent Student's t-test and one-way ANOVA with Student-Newman-Keuls post hoc test were performed to compare the differences between groups. Spearman correlation analysis was used to examine the correlation between miR-539 and IGF-1R mRNA in PDAC tissues. $\mathrm{P}<0.05$ was considered statistically significant.

\section{Results}

miR-539 is significantly downregulated in PDAC tissues and cell lines. To investigate the expression pattern of miR-539 in PDAC, we measured the miR-539 expression in 45 pairs of PDAC tissues and adjacent normal pancreatic tissues using RT-qPCR. Our data showed that the miR-539 expression obviously decreased in PDAC tissues compared with that in adjacent normal pancreatic tissues (Fig. 1A, $\mathrm{P}<0.05)$. The association between this expression and the clinicopathological characteristics of PDAC was evaluated to determine the clinical value of miR-539 in PDAC. Table I shows that the expression level of miR-539 was significantly associated with TNM stage $(\mathrm{P}=0.011)$ and lymph node metastasis $(\mathrm{P}=0.026)$. However, no correlation was observed between miR-539 and other clinicopathological features, including age, sex, tumour site and tumour differentiation (all P>0.05). RT-qPCR analysis was performed to quantify the miR-539 expression in four PDAC cell lines, including
Table I. Association between miR-539 expression and clinicopathological characteristics of patients with pancreatic ductal adenocarcinoma.

\begin{tabular}{|c|c|c|c|}
\hline \multirow{2}{*}{$\begin{array}{l}\text { Clinicopathological } \\
\text { characteristics }\end{array}$} & \multicolumn{2}{|c|}{$\begin{array}{l}\text { miR-539 } \\
\text { expression }\end{array}$} & \multirow[b]{2}{*}{ P-value } \\
\hline & Low & High & \\
\hline Age & & & 0.273 \\
\hline$<60$ years & 11 & 7 & \\
\hline$\geq 60$ years & 12 & 15 & \\
\hline Sex & & & 0.465 \\
\hline Male & 15 & 12 & \\
\hline Female & 8 & 10 & \\
\hline Tumour size & & & 0.661 \\
\hline$<2 \mathrm{~cm}$ & 10 & 11 & \\
\hline$\geq 2 \mathrm{~cm}$ & 13 & 11 & \\
\hline Tumour differentiation & & & 0.449 \\
\hline Well & 11 & 13 & \\
\hline Poor & 12 & 9 & \\
\hline TNM stage & & & $0.011^{\mathrm{a}}$ \\
\hline I-II & 5 & 13 & \\
\hline III-IV & 18 & 9 & \\
\hline Lymph node metastasis & & & $0.026^{\mathrm{a}}$ \\
\hline Negative & 7 & 14 & \\
\hline Positive & 16 & 8 & \\
\hline
\end{tabular}

${ }^{\mathrm{a}} \mathrm{P}<0.05$.

Sw1990, Panc-1, Bxpc-3 and Aspc-1. The expression level of miR-539 was underexpressed in all four PDAC cell lines compared with that of the normal human pancreatic cell line HPDE6c7 $(\mathrm{P}<0.05$; Fig. 1B). These results suggested that miR-539 downregulation might be correlated with PDAC progression.

miR-539 plays a negative role in human PDAC cell proliferation, colony formation and invasion. To evaluate the effect of miR-539 on the oncogenic phenotype of PDAC, we upregulated the miR-539 expression in Sw1990 and Bxpc-3 cells, whose endogenous miR-539 expression was relatively lower among that of the four PDAC cell lines ( $\mathrm{P}<0.05$; Fig. 2A). The effect of miR-539 overexpression on PDAC cell proliferation was examined with a CCK-8 assay. We found that miR-539 upregulation reduced Sw1990 and Bxpc-3 cell proliferation $(\mathrm{P}<0.05$; Fig. 2B). A colony formation assay was further conducted to confirm the inhibitory effect of miR-539 on PDAC cell proliferation. In Fig. 2C, the upregulation of miR-539 caused a significant decrease in the colony formation of Sw1990 and Bxpc-3 cells $(\mathrm{P}<0.05)$. We next explored the effect of miR-539 on the cell invasion ability of PDAC. The results of cell invasion assay revealed that miR-539 overexpression decreased the invasion capacities of Sw1990 and Bxpc-3 cells $(\mathrm{P}<0.05$; Fig. 2D). These results suggested that miR-539 could perform tumour suppressive roles in PDAC. 

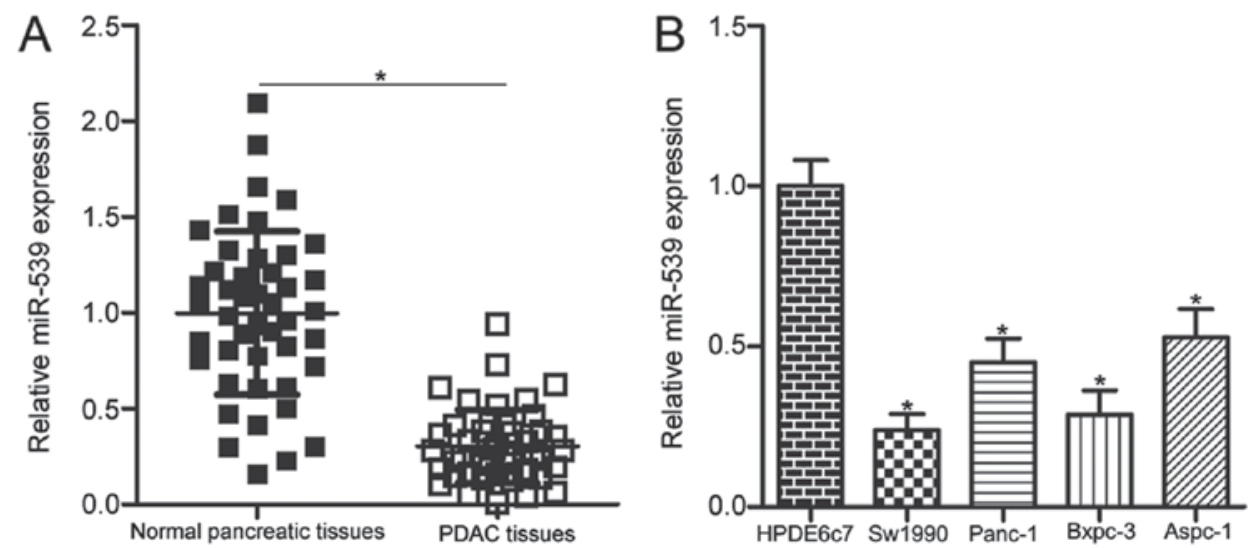

Figure 1. miR-539 expression levels in PDAC tissues and cell lines. (A) RT-qPCR was used to detect the miR-539 level in 45 pairs of PDAC tissues and adjacent normal pancreatic tissues. "P<0.05 vs. normal pancreatic tissues. (B) miR-539 expression was determined in four human PDAC cell lines (Sw1990, Panc-1, Bxpc-3 and Aspc-1) and a normal human pancreatic cell line (HPDE6c7). "P<0.05 vs. HPDE6c7. PDAC, pancreatic ductal adenocarcinoma; RT-qPCR, reverse transcription-quantitative polymerase chain reaction.
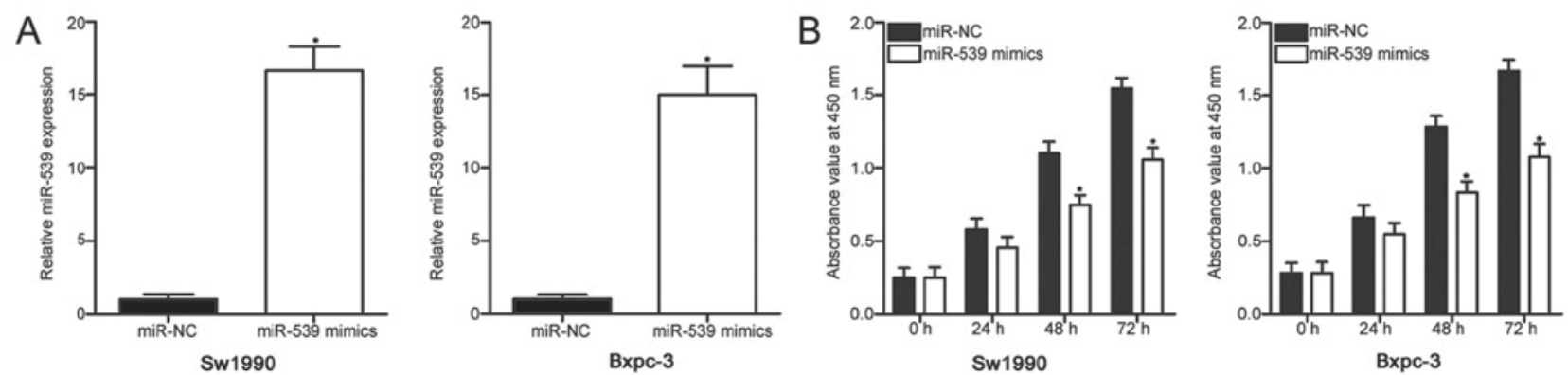

C
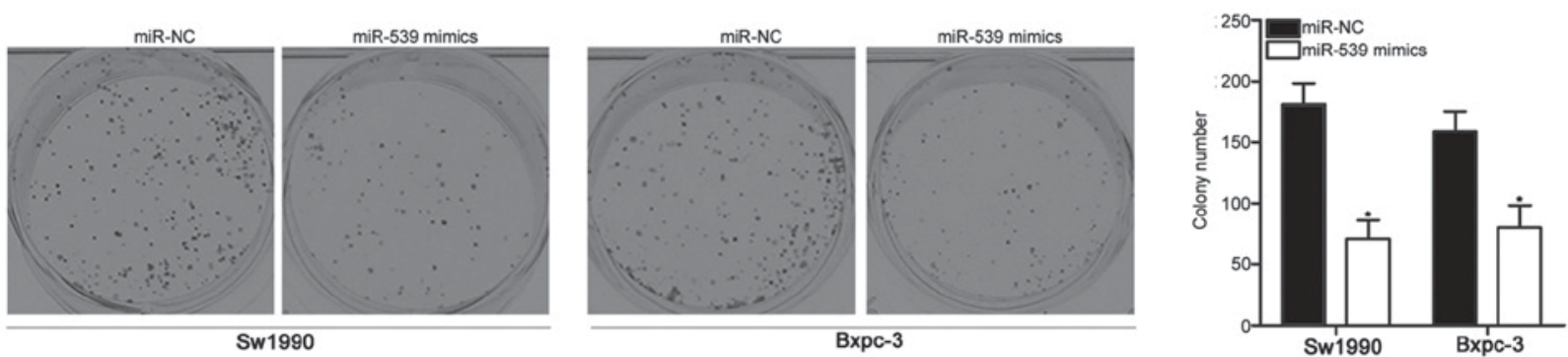

D
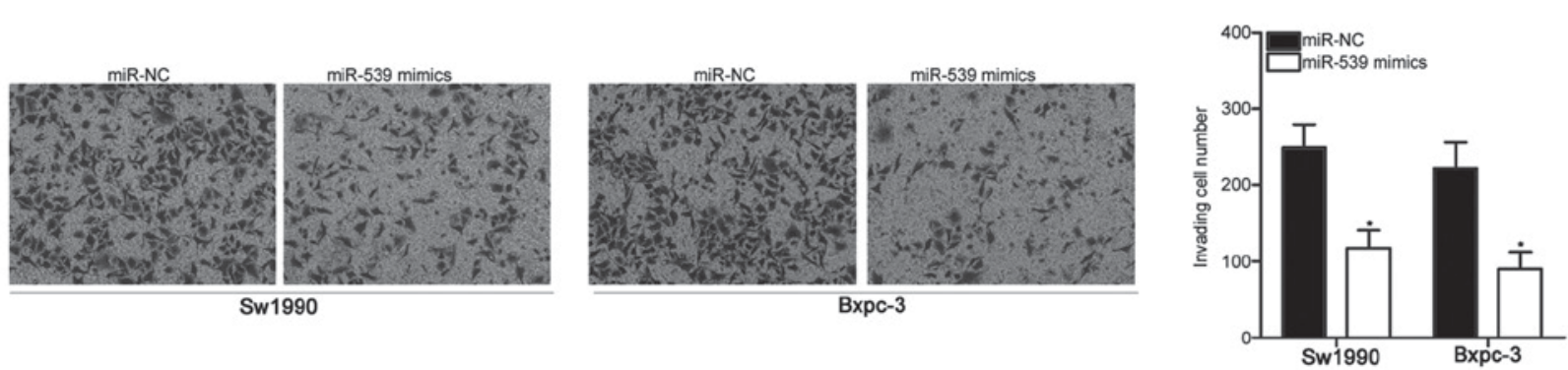

Figure 2. miR-539 upregulation represses the proliferation, colony formation and invasion of Sw1990 and Bxpc-3 cells. Sw1990 and Bxpc-3 cells were transfected with miR-539 mimics or miR-NC and used in the following assays. (A) Transfection efficiency was evaluated through RT-qPCR. ${ }^{\mathrm{P}}<0.05$ vs. miR-NC. (B) Cell proliferative ability was examined with CCK- 8 assay. ${ }^{*} \mathrm{P}<0.05$ vs. miR-NC. (C) Colony formation assay was performed to analyse the colony formation ability. The number of cell colonies was counted under an inverted microscope. $\mathrm{P}<0.05$ vs. miR-NC. (D) Invasion ability was determined using a cell invasion assay. The invading cells were photographed and counted under an inverted microscope at a magnification of x 200 . "P<0.05 vs. miR-NC. NC, negative control; CCK-8, Cell Counting Kit-8; RT-qPCR, reverse transcription-quantitative polymerase chain reaction.

$I G F-1 R$ is a direct target of $m i R-539$ in PDAC. To elucidate the mechanisms by which miR-539 executed its inhibitory effects on PDAC cells, we predicted the putative targets of miR-539 through bioinformatics prediction. IGF-1R (Fig. 3A), a well-known oncogene in PDAC (21-27), was predicted as a major highly conserved target of miR-539 and was selected for further analysis. Luciferase reporter assay was performed to validate this speculation and to investigate whether miR-539 could directly interact with the 3'-UTR of IGF-1R. miR-539 mimics or miR-NC was transfected into 


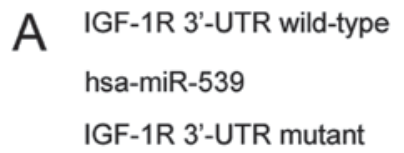
A IGF-1R 3'-UTR wild-type
hsa-miR-539
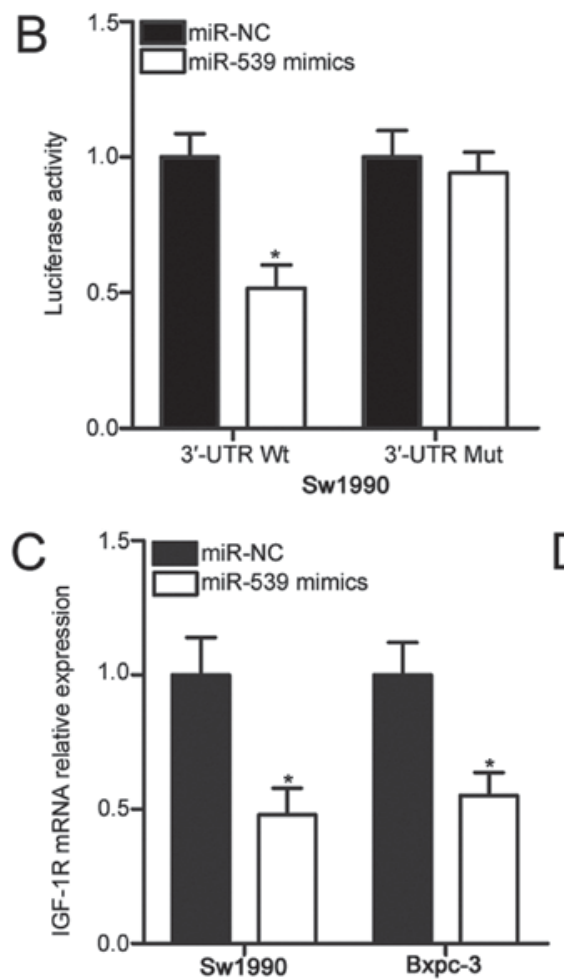
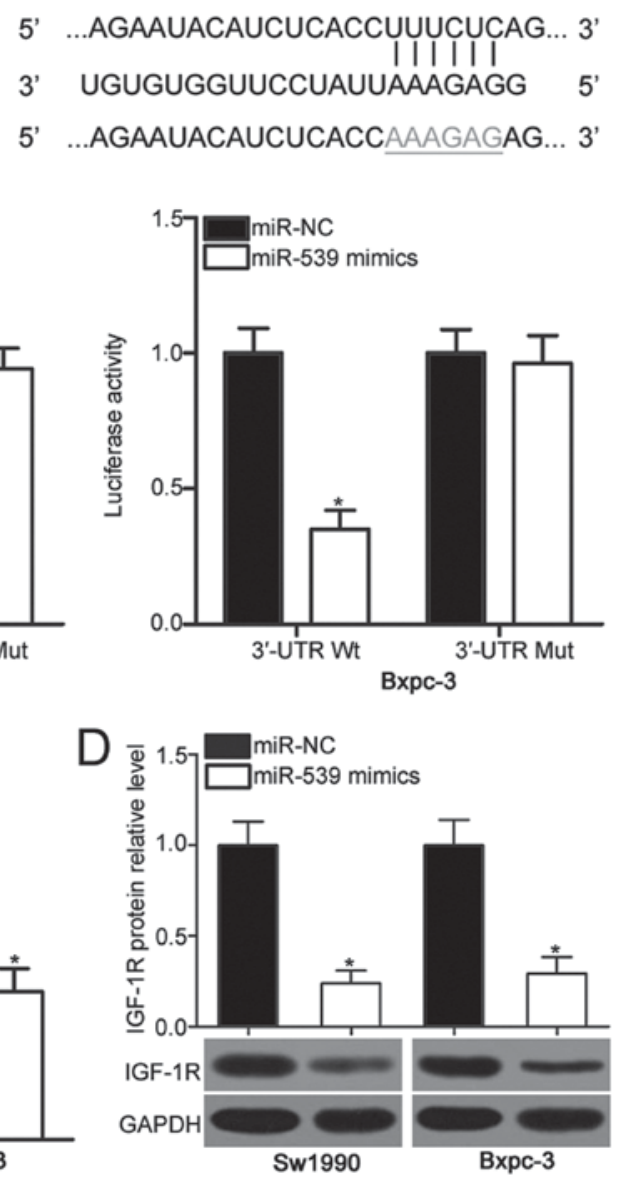

Figure 3. IGF-1R is a direct target gene of miR-539 in PDAC. (A) Predicted binding sites of miR-539 in the 3'-UTR of IGF-1R and its mutant control. (B) Luciferase activities were measured in Sw1990 and Bxpc-3 cells transfected with miR-539 mimics or miR-NC together with pMIR-IGF-1R-3'-UTR Wt or pMIR-IGF-1R-3'-UTR Mut. ${ }^{*}<0.05$ vs. miR-NC. (C and D) RT-qPCR and western blot analysis of the mRNA and protein expression levels of IGF-1R in Sw1990 and Bxpc-3 cells transfected with miR-539 mimics or miR-NC, respectively. * $<<0.05$ vs. miR-NC. IGF-1R, insulin-like growth factor 1 receptor; PDAC, pancreatic ductal adenocarcinoma; 3'-UTR; 3'-untranslated region; RT-qPCR, reverse transcription-quantitative polymerase chain reaction; NC, negative control.

Sw1990 and Bxpc-3 cells in combination with pMIR-Report vector containing wild-type (Wt) IGF-1R 3'-UTR or mutant (Mut) IGF-1R 3'-UTR. The ectopic of miR-539 expression resulted in a significant decrease in the luciferase activities of pMIR-IGF-1R-3'-UTR Wt $(\mathrm{P}<0.05)$. However, the mutation of the binding sequences of miR-539 in the 3'-UTR of IGF-1R abolished the suppressive effect of miR-539 on luciferase activities (Fig. 3B). We also examined the effect of miR-539 on the endogenous IGF-1R expression in PDAC. RT-qPCR and Western blot analysis demonstrated that the enforced expression of miR-539 reduced the IGF-1R expression in Sw1990 and Bxpc-3 cells at mRNA $(\mathrm{P}<0.05$; Fig. $3 \mathrm{C})$ and protein $(\mathrm{P}<0.05$; Fig. 3D) levels. These results evidently suggested that IGF-1R is a direct target of miR-539 in PDAC.

IGF-1R upregulation in PDAC tissues is inversely correlated with miR-539 level. RT-qPCR analysis was carried out on 45 pairs of PDAC tissues and adjacent normal pancreatic tissues to further examine the association between miR-539 and IGF-1R in PDAC. The mRNA expression level of IGF-1R was remarkably overexpressed in the PDAC tissues compared with that in the adjacent normal pancreatic tissues $(\mathrm{P}<0.05$; Fig. 4A). Western blot analysis also revealed that PDAC tissues exhibited a significantly upregulated protein level of
IGF-1R compared with that in the adjacent normal pancreatic tissues (Fig. 4B). Furthermore, the association between the mRNA expression of IGF-1R and miR-539 levels in the PDAC tissues was examined through Spearman correlation analysis. In Fig. 4C, the mRNA expression of IGF-1R was inversely associated with miR-539 expression levels in the PDAC tissues $(r=-0.5841, P<0.0001)$. These results suggested that the miR-539 downregulation might at least partly increase the IGF-1R expression in PDAC tissues.

Recovered IGF-1R expression partially counteracts the suppressive effects of miR-539 overexpression on PDAC cells. A series of rescue experiments were applied to further address that the tumor suppressive roles of miR-539 on PDAC cells were mediated by the inhibition of IGF-1R. Sw1990 and Bxpc-3 cells were cotransfected with miR-539 mimics and empty pCMV vector or IGF-1R overexpression vector (pCMV-IGF-1R). After transfection for $72 \mathrm{~h}$, western blot analysis confirmed that the IGF-1R protein levels were restored in Sw1990 and Bxpc-3 cells cotransfected with miR-539 mimics and pCMV-IGF-1R compared with those in the cells cotransfected with miR-539 mimics and pCMV $(\mathrm{P}<0.05$; Fig. 5A). CCK-8, colony formation and cell invasion assays indicated that the IGF-1R overexpression partially rescued the 

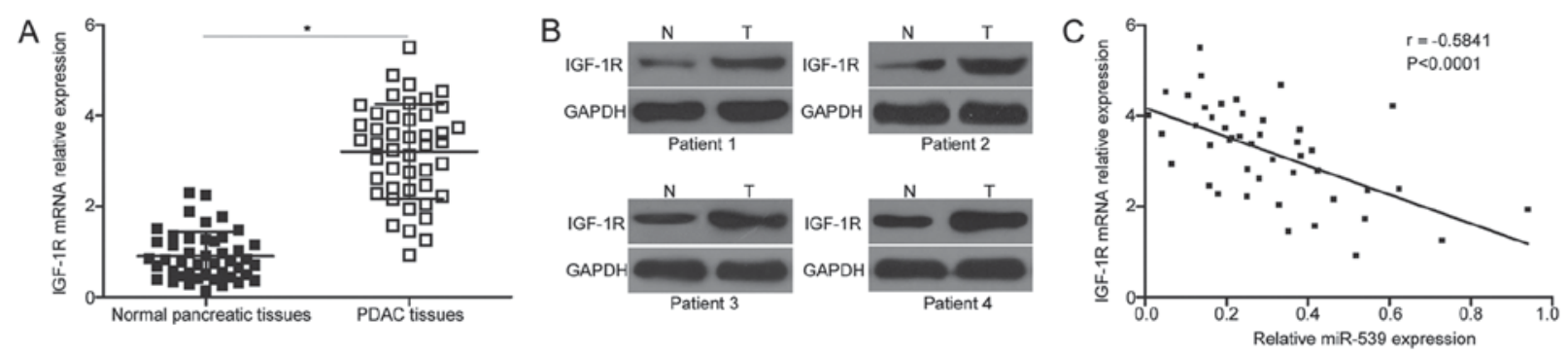

Figure 4. IGF-1R overexpression is negatively correlated with the miR-539 level in PDAC tissues. (A and B) mRNA and protein expression levels of IGF-1R measured in PDAC tissues and adjacent normal pancreatic tissues through RT-qPCR and western blot analysis, respectively. "P<0.05 vs. normal pancreatic tissues as indicated. (C) Spearman correlation analysis of the association between miR-539 and mRNA level of IGF-1R in PDAC tissues. $r=-0.5841, \mathrm{P}<0.0001$. IGF-1R, insulin-like growth factor 1 receptor; PDAC, pancreatic ductal adenocarcinoma; RT-qPCR, reverse transcription-quantitative polymerase chain reaction.

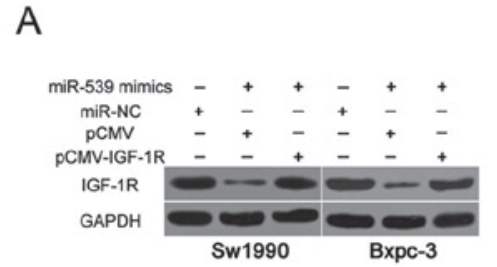

C

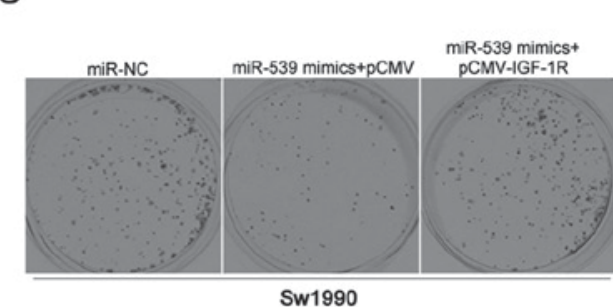

D

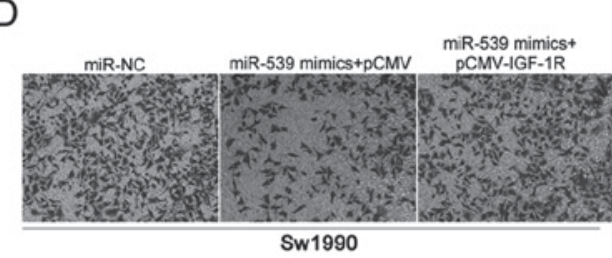

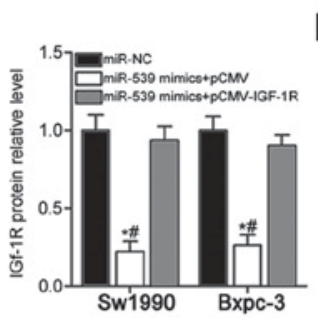

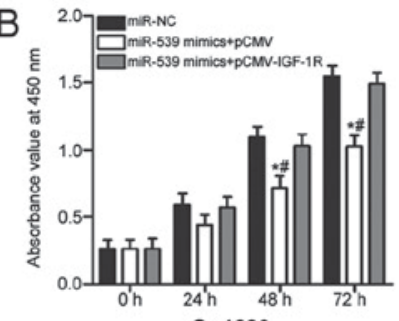

Sw1990

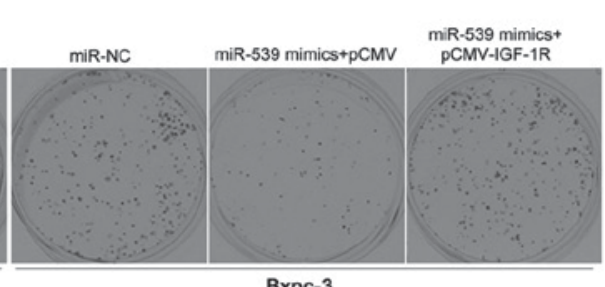

Bxpc-3

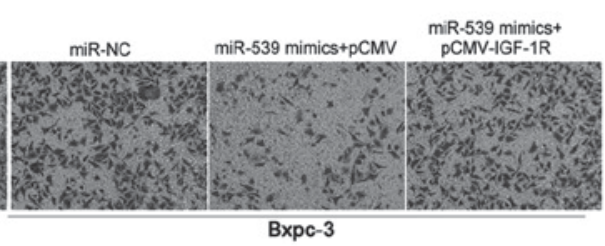

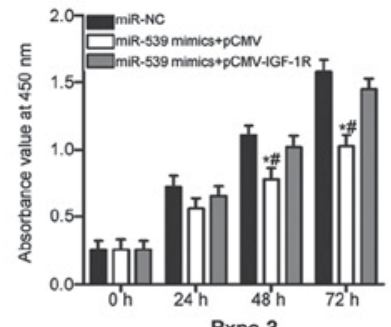

Bxpc-3
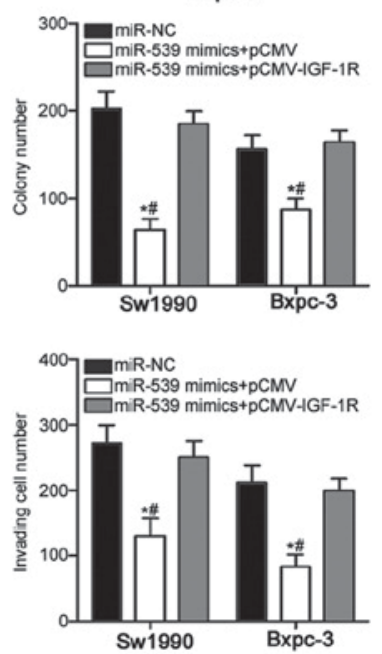

Figure 5. IGF-1R overexpression partially rescues the effects of miR-539 overexpression on the proliferation, colony formation and invasion of Sw1990 and Bxpc-3 cells. Sw1990 and Bxpc-3 cells were transfected with miR-539 mimics in combination with pCMV or pCMV-IGF-1R and subjected to the following assays. (A) Western blot analysis was performed to quantify the protein level of IGF-1R. ${ }^{*} \mathrm{P}<0.05$ vs. miR-NC. "P<0.05 vs. miR-539 mimics+pCMV-IGF-1R. (B-D) CCK-8, colony formation and cell invasion assays were applied to determine cell proliferation, colony formation and invasion ability, respectively. magnification, $\mathrm{x} 200$. "P<0.05 vs. miR-NC. ${ }^{\text {PP}}<0.05$ vs. miR-539 mimics+pCMV-IGF-1R. IGF-1R, insulin-like growth factor 1 receptor; NC, negative control; CCK-8, Cell Counting Kit-8.

inhibitory effects of the miR-539 overexpression on Sw1990 and Bxpc-3 cell proliferation $(\mathrm{P}<0.05$; Fig. $5 \mathrm{~B})$, colony formation $(\mathrm{P}<0.05$; Fig. 5C) and invasion $(\mathrm{P}<0.05$; Fig. 5D). Overall, the tumour-suppressing effects of miR-539 overexpression on PDAC cells are partly achieved by downregulating the IGF-1R expression.

\section{Discussion}

miRNAs possess the oncogenic and tumour-suppressive roles in the carcinogenesis and progression of PDAC by regulating the expression of numerous cancer-related genes (28-30). Thus, the investigation on the expression and roles of miRNAs in PDAC may promote the identification of novel and effective targets for the clinical diagnosis and treatment of patients with this cancer. This study is the first to explore the expression pattern, biological roles and underlying mechanisms of miR-539 in PDAC. Here, we found that miR-539 was obviously downregulated in the PDAC tissues and cell lines. The decreased miR-539 level was strongly correlated with TNM stage and lymph node metastasis. Functional experiments indicated that the upregulation of miR-539 restricted the cell proliferation, colony formation and invasion of PDAC. Furthermore, IGF-1R was confirmed as a direct target of miR-539 in PDAC. IGF-1R upregulation in the PDAC tissues was inversely correlated with the miR-539 level. IGF-1R 
overexpression partially rescued the suppressive roles in the PDAC cells induced by miR-539 overexpression. These results suggested that miR-539 might be a suitable therapeutic target for the treatment of patients with this fatal malignancy.

miR-539 dysregulation has been observed in multiple types of human cancer. For example, miR-539 is downregulated in colorectal cancer tissues, and this phenomenon is associated with clinical stage and lymph node metastasis (17). The expression pattern of miR-539 is also decreased in glioma (18), oesophageal cancer (19), hepatocellular carcinoma $(31,32)$, prostate cancer (33), nasopharyngeal carcinoma (34), osteosarcoma (35) and thyroid cancer (36). These findings suggested that miR-539 downregulation is a common event in human cancer and may represent useful markers for cancer diagnosis.

miR-539 performs important functional roles in tumour occurrence and development. For instance, miR-539 re-expression suppresses the growth and metastasis of colorectal cancer cells in vitro and impairs tumour growth in vivo (17). Quan et al (18) found that ectopic miR-539 expression causes an evident reduction in the cell proliferation and invasion of glioma. Li et al (19) reported that the ectopic of miR-539 expression represses the epithelial-to-mesenchymal transition of cells in oesophageal cancer. Zhu (31) and Liu et al (32) demonstrated that miR-539 upregulation restricts the growth and metastasis of hepatocellular carcinoma cells, induces apoptosis in vitro, decreases tumour growth and tumourigenesis in vivo and increases the chemosensitivity of trioxide-resistant cells to arsenic trioxide. Zhang et al (33) revealed that miR-539 overexpression inhibits the proliferation, migration and invasion of prostate cancer cells in vitro and in vivo. Lv et al indicated that the induced miR-539 expression attenuates the proliferation of nasopharyngeal carcinoma cells, triggers cell cycle arrest in vitro and reduces cell growth in vivo (34). Jin and Wang (35) and Gu and Sun (36) showed that the resumption of miR-539 expression prevents the migration and invasion of osteosarcoma and thyroid cancer cells. These findings suggested that exogenous miR-539 may have a therapeutic value for patients with cancer.

miRNAs can affect carcinogenesis and cancer progression by directly regulating target genes expression. Several targets identified for miR-539 thus far include RUNX2 (17) in colorectal cancer, DIXDC1 (18) in glioma, TWIST1 (19) in esophageal cancer, FSCN1 (32) in hepatocellular carcinoma, SPAG5 (33) in prostate cancer, CDK4 (34) in nasopharyngeal carcinoma, MMP8 (35) in osteosarcoma and CARMA1 (36) in thyroid cancer. In our study, IGF-1R, a transmembrane tyrosine kinase receptor, was validated as a novel target of miR-539 in PDAC. IGF-1R protein is composed of two extracellular $\alpha$ subunits with a ligand-binding site and two transmembrane $\beta$ subunits with intracellular tyrosine kinase activity (37). IGF-1R is overexpressed in numerous kinds of human malignancies, such as ovarian cancer (38), hepatocellular carcinoma (39), renal cell carcinoma (40), lung cancer (41), gastric cancer (42) and bladder cancer (43). IGF-1R is also highly expressed in PDAC tissues, and this upregulation is strongly associated with tumour location, histological grade and TNM stage $(21,22)$. The prognosis of patients with PDAC with a high IGF-1R expression is poorer than that of patients with a low IGF-1R expression (22). IGF-1R dysregulation is implicated in the aggressiveness of PDAC by regulating various pathological processes, such as cell proliferation, apoptosis, migration, invasion, epithelial-to-mesenchymal transition, angiogenesis and chemoresistance (23-27). Thus, the inhibition of IGF-1R is a promising therapeutic strategy for patients with PDAC.

In conclusion, this study demonstrated that the miR-539 expression was downregulated in PDAC tissues and cell lines. Decreased miR-539 levels were significantly correlated with TNM stage and lymph node metastasis. miR-539 upregulation restricted the proliferation, colony foramtion and invasion of PDAC cells by directly targeting and inhibiting IGF-1R. These findings may provide novel insights into the mechanisms associated with the rapid growth and early metastasis of PDAC.

\section{Acknowledgements}

Not applicable.

\section{Funding}

No funding was received.

\section{Availability of data and materials}

The datasets used and/or analyzed during the present study are available from the corresponding author on reasonable request.

\section{Authors' contributions}

SL and YL designed the research. YL, LR, JZ, and RL performed functional experiments. All authors read and approved the final draft.

\section{Ethics approval and consent to participate}

The present study was approved by the Ethics Committee of Yidu Central Hospital of Weifang, and was performed in accordance with the Declaration of Helsinki and the guidelines of the Ethics Committee of Yidu Central Hospital of Weifang. Written informed consent was obtained from all patients for the use of their clinical tissues.

\section{Consent for publication}

Not applicable.

\section{Competing interests}

The authors declare that they have no competing interests.

\section{References}

1. Torre LA, Bray F, Siegel RL, Ferlay J, Lortet-Tieulent J and Jemal A: Global cancer statistics, 2012. CA Cancer J Clin 65: 87-108, 2015.

2. Modolell I, Guarner L and Malagelada JR: Vagaries of clinical presentation of pancreatic and biliary tract cancer. Ann Oncol 10 (Suppl 4): 82-84, 1999.

3. Jiao F, Hu H, Yuan C, Wang L, Jiang W, Jin Z, Guo Z and Wang L: Elevated expression level of long noncoding RNA MALAT-1 facilitates cell growth, migration and invasion in pancreatic cancer. Oncol Rep 32: 2485-2492, 2014.

4. Maitra A and Hruban RH: Pancreatic cancer. Annu Rev Pathol 3: 157-188, 2008 
5. Moir J, White SA, French JJ, Littler P and Manas DM: Systematic review of irreversible electroporation in the treatment of advanced pancreatic cancer. Eur J Surg Oncol 40: 1598-1604, 2014.

6. Burkey MD, Feirman S, Wang H, Choudhury SR, Grover S and Johnston FM: The association between smokeless tobacco use and pancreatic adenocarcinoma: A systematic review. Cancer Epidemiol 38: 647-653, 2014.

7. Bartel DP: MicroRNAs: Genomics, biogenesis, mechanism, and function. Cell 116: 281-297, 2004.

8. Bartel DP: MicroRNAs: Target recognition and regulatory functions. Cell 136: 215-233, 2009.

9. Fan Y, Shi C, Li T and Kuang T: MicroRNA-454 shows anti-angiogenic and anti-metastatic activity in pancreatic ductal adenocarcinoma by targeting LRP6. Am J Cancer Res 7: 139-147, 2017.

10. Zhang G, Ai D, Yang X, Ji S, Wang Z and Feng S: MicroRNA-610 inhibits tumor growth of melanoma by targeting LRP6. Oncotarget 8: 97361-97370, 2017.

11. Jin Y, Tao LP, Yao SC, Huang QK, Chen ZF, Sun YJ and Jin SQ MicroRNA-582-5p suppressed gastric cancer cell proliferation via targeting AKT3. Eur Rev Med Pharmacol Sci 21: 5112-5120, 2017.

12. Wang X and Wu X: The Role of MicroRNA-1207-5p in colorectal cancer. Clin Lab 63: 1875-1882, 2017.

13. Yang D, Du G, Xu A, Xi X and Li D: Expression of miR-149-3p inhibits proliferation, migration, and invasion of bladder cancer by targeting S100A4. Am J Cancer Res 7: 2209-2219, 2017.

14. Liu S, Liu K, Zhang W, Wang Y, Jin Z, Jia B and Liu Y: miR-449a inhibits proliferation and invasion by regulating ADAM10 in hepatocellular carcinoma. Am J Transl Res 8: 2609-2619, 2016.

15. Cheng RF, Wang J, Zhang JY, Sun L, Zhao YR, Qiu ZQ, Sun BC and Sun Y: MicroRNA-506 is up-regulated in the development of pancreatic ductal adenocarcinoma and is associated with attenuated disease progression. Chin J Cancer 35: 64, 2016.

16. Tu MJ, Pan YZ, Qiu JX, Kim EJ and Yu AM: MicroRNA-1291 targets the FOXA2-AGR2 pathway to suppress pancreatic cancer cell proliferation and tumorigenesis. Oncotarget 7: 45547-45561, 2016.

17. Wen D, Li S, Jiang W, Zhu J, Liu J and Zhao S: miR-539 inhibits human colorectal cancer progression by targeting RUNX2. Biomed Pharmacother 95: 1314-1320, 2017

18. Quan J, Qu J and Zhou L: MicroRNA-539 inhibits glioma cell proliferation and invasion by targeting DIXDC1. Biomed Pharmacother 93: 746-753, 2017

19. Li S, Yang F, Wang M, Cao W and Yang Z: miR-378 functions as an onco-miRNA by targeting the ST7L/Wnt/ $\beta$-catenin pathway in cervical cancer. Int J Mol Med 40: 1047-1056, 2017.

20. Livak KJ and Schmittgen TD: Analysis of relative gene expression data using real-time quantitative PCR and the 2(-Delta Delta C(T)) method. Methods 25: 402-408, 2001.

21. Xu JW, Wang TX, You L, Zheng LF, Shu H, Zhang TP and Zhao YP: Insulin-like growth factor 1 receptor (IGF-1R) as a target of MiR-497 and plasma IGF-1R levels associated with TNM stage of pancreatic cancer. PLoS One 9: e92847, 2014.

22. Hirakawa T, Yashiro M, Murata A, Hirata K, Kimura K, Amano R, Yamada N, Nakata B and Hirakawa K: IGF-1 receptor and IGF binding protein-3 might predict prognosis of patients with resectable pancreatic cancer. BMC Cancer 13: 392, 2013.

23. Subramani R, Lopez-Valdez R, Arumugam A, Nandy S, Boopalan $\mathrm{T}$ and Lakshmanaswamy R: Targeting insulin-like growth factor 1 receptor inhibits pancreatic cancer growth and metastasis. PLoS One 9: e97016, 2014.

24. Zheng D, Golubovskaya V, Kurenova E, Wood C, Massoll NA, Ostrov D, Cance WG and Hochwald SN: A novel strategy to inhibit FAK and IGF-1R decreases growth of pancreatic cancer xenografts. Mol Carcinog 49: 200-209, 2010.

25. Farhana L, Dawson MI, Murshed F, Das JK, Rishi AK and Fontana JA: Upregulation of miR-150* and miR-630 induces apoptosis in pancreatic cancer cells by targeting IGF-1R. PLoS One 8: e61015, 2013
26. Tian X, Hao K, Qin C, Xie K, Xie X and Yang Y: Insulin-like growth factor 1 receptor promotes the growth and chemoresistance of pancreatic cancer. Dig Dis Sci 58: 2705-2712, 2013.

27. Moser C, Schachtschneider P, Lang SA, Gaumann A, Mori A, Zimmermann J, Schlitt HJ, Geissler EK and Stoeltzing O: Inhibition of insulin-like growth factor-I receptor (IGF-IR) using NVP-AEW541, a small molecule kinase inhibitor, reduces orthotopic pancreatic cancer growth and angiogenesis. Eur J Cancer 44: 1577-1586, 2008.

28. Qadir MI and Faheem A: miRNA: A diagnostic and therapeutic tool for pancreatic cancer. Crit Rev Eukaryot Gene Expr 27: 197-204, 2017.

29. Liang S, Gong X, Zhang G, Huang G, Lu Y and Li Y: MicroRNA-140 regulates cell growth and invasion in pancreatic duct adenocarcinoma by targeting iASPP. Acta Biochim Biophys Sin (Shanghai) 48: 174-181, 2016.

30. Keklikoglou I, Hosaka K, Bender C, Bott A, Koerner C, Mitra D, Will R, Woerner A, Muenstermann E1 and Wilhelm H, et al: MicroRNA-206 functions as a pleiotropic modulator of cell proliferation, invasion and lymphangiogenesis in pancreatic adenocarcinoma by targeting ANXA2 and KRAS genes. Oncogene 34: 4867-4878, 2015.

31. Zhu C, Zhou R, Zhou Q, Chang Y and Jiang M: microRNA-539 suppresses tumor growth and tumorigenesis and overcomes arsenic trioxide resistance in hepatocellular carcinoma. Life Sci 166: 34-40, 2016.

32. Liu Y, Hong W, Zhou C, Jiang Z, Wang G, Wei G and Li X: miR-539 inhibits FSCN1 expression and suppresses hepatocellular carcinoma migration and invasion. Oncol Rep 37: 2593-2602, 2017.

33. Zhang H, Li S, Yang X, Qiao B, Zhang Z and Xu Y: miR-539 inhibits prostate cancer progression by directly targeting SPAG5. J Exp Clin Cancer Res 35: 60, 2016.

34. Lv LY, Wang YZ, Zhang Q, Zang HR and Wang XJ: miR-539 induces cell cycle arrest in nasopharyngeal carcinoma by targeting cyclin-dependent kinase 4. Cell Biochem Funct 33: $534-540,2015$.

35. Jin $\mathrm{H}$ and Wang W: MicroRNA-539 suppresses osteosarcoma cell invasion and migration in vitro and targeting Matrix metallopeptidase-8. Int J Clin Exp Pathol 8: 8075-8082, 2015.

36. Gu L and Sun W: MiR-539 inhibits thyroid cancer cell migration and invasion by directly targeting CARMA1. Biochem Biophys Res Commun 464: 1128-1133, 2015.

37. Hu Q, Gong JP, Li J, Zhong SL, Chen WX, Zhang JY, Ma TF, Ji H, Lv MM, Zhao JH and Tang JH: Down-regulation of miRNA-452 is associated with adriamycin-resistance in breast cancer cells. Asian Pac J Cancer Prev 15: 5137-5142, 2014.

38. An Y, Cai L, Wang Y, Zhu D, Guan Y and Zheng J: Onkologie. Onkologie 32: 638-644, 2009.

39. E C, Li J, Shao D, Zhang D, Pan Y, Chen L and Zhang X: The insulin-like growth factor-I receptor inhibitor picropodophyllin-induced selective apoptosis of hepatocellular carcinoma cell through a caspase-dependent mitochondrial pathway. Oncol Res 21: 103-110, 2013

40. Sichani MM, Yazdi FS, Moghaddam NA, Chehrei A, Kabiri M, Naeimi A and Taheri D: Prognostic value of insulin-like growth factor-I receptor expression in renal cell carcinoma. Saudi J Kidney Dis Transpl 21: 69-74, 2010.

41. Zhao S, Qiu Z, He J, Li L and Li W: Insulin-like growth factor receptor 1 (IGF1R) expression and survival in non-small cell lung cancer patients: A meta-analysis. Int J Clin Exp Pathol 7: 6694-6704, 2014

42. Gryko M, Kiśluk J, Cepowicz D, Zińczuk J, Kamocki Z, Guzińska-Ustymowicz K, Pryczynicz A, Czyżewska J, Kemona A and Kędra B: Expression of insulin-like growth factor receptor type 1 correlate with lymphatic metastases in human gastric cancer. Pol J Pathol 65: 135-140, 2014.

43. Xie QX, Lin XC, Zhang MF, Han CX and Guo YH: Expression of IGF-I and IGF-IR in bladder cancer. Ai Zheng 23: 707-709, 2004 (In Chinese). 